\title{
Simultaneous Discrimination of Speed is More Difficult than Simultaneous Discrimination of Size for Both Pigeons and People
}

\author{
Olga F. Lazareva* and Rebecca Share
}

Drake University, Des Moines, Iowa, USA

*Corresponding author (Email: olga.lazareva@drake.edu)

Citation - Lazareva, O. F., \& Share, R. (2017). Simultaneous discrimination of speed is more difficult than simultaneous discrimination of size for both pigeons and people. Animal Behavior and Cognition, 4(3), 252-266. https://doi.org/10.26451/abc.04.03.05.2017

\begin{abstract}
Previous reports suggest that pigeons are highly sensitive to speed of motion (Cook, Beale, Koban, 2011; Herbranson, Fremouw, \& Shimp, 2002). In contrast, we found that pigeons required more extensive training to learn speed discrimination than size discrimination (Lazareva, Young, \& Wasserman, 2014). However, our results were based on a comparison of two experiments conducted in different laboratories, complicating the interpretation of the data. Here, we trained pigeons to perform size discrimination or speed discrimination in a two-alternative simultaneous discrimination task using a within-subject design. All birds acquired size discrimination much faster than speed discrimination, confirming our prior report. We further explored pigeons' sensitivity to differences in speed and size by training them to discriminate two end-point stimuli in a two-alternative forced-choice task and then presenting a wide range of testing stimuli located between the training end-point stimuli. The results again indicated weaker control by the differences in speed in comparison to size; comparable results were obtained for human participants.
\end{abstract}

Keywords - Speed, Size, Simultaneous Discrimination, Pigeons, Sensitivity, Forced Choice

The ability to discriminate between different sizes and different velocities is important for many organisms. For example, pigeons would benefit from discriminating and preferring a larger piece of food instead of a smaller piece of food. Similarly, a predator that moves slowly, such as a gliding red-tailed hawk, poses less of a threat to a pigeon than the same hawk diving from above. People too benefit from discriminating smaller from larger sizes and slower from faster objects when they select appropriately sized boxes to pack or determine whether to pass a car on a highway.

Multiple studies have shown that pigeons are sensitive to relatively minor differences in sizes. For example, pigeons trained to perform a matching-to-sample task using a circle and a triangle as stimuli showed significant decrement in their discriminative performance when the size of the stimuli was reduced by as little as 33\% (Pisacreta, Potter, \& Lefave, 1984). Similar results were obtained in a go/nogo procedure, in which a $22 \%$ increase or decrease in a size of a discriminative stimulus produced a noticeable generalization decrement (Jenkins, Pascal, \& Walker, 1958; see also Wildemann \& Holland, 1973). More recently, pigeons were reported to exhibit similar performance impairment in a fouralternative forced-choice task when the discriminative stimuli (line drawings and shaded threedimensional objects) were resized (Peissig, Kirkpatrick-Steger, Young, Wasserman, \& Biederman, 2006). 
Pigeons are also sensitive to differences in object velocities. In a representative experiment, pigeons were trained to discriminate an object rotating in depth slowly $\left(55^{\circ}\right.$ per second) or quickly $\left(200^{\circ}\right.$ per second) in a go/no-go task (Cook, Beale, \& Koban, 2011). Pigeons' discriminative performance appeared to be impressively robust: The objects rotating at $20^{\circ}, 30^{\circ}, 66.7^{\circ}$, and $85.7^{\circ}$ per second were still classified as "slow", with a generalization decrement observed for $85.7^{\circ}$ per second. Pigeons were also able to transfer this speed discrimination to a speed of rotation along a z-axis and to a speed of moving along a complex path, suggesting that pigeons were able to extract and apply a general concept of a velocity of an object. Similarly, pigeons can be trained to classify objects on the basis of the speed of their vertical motion (Herbranson, Fremouw, \& Shimp, 2002), direction of rotation (Koban \& Cook, 2009), or type of biological motion (Asen \& Cook, 2012). In all of these studies, motion-based discrimination appeared to be a relatively easy task, sometimes acquired in as little as 500 trials.

In contrast to these reports, Lazareva, Young, and Wasserman (2014) found that pigeons required more than 2,500 trials to learn a simultaneous two-alternative discrimination of two objects moving along $\mathrm{y}$-axis at different speeds; in comparison, a simultaneous two-alternative discrimination of size has been mastered in as little as 360 trials. Because this result appeared to be at odds with the existing literature, we conducted a series of experiments systematically exploring pigeons' ability to discriminate differences in size and speed. In Experiment 1, we attempted to train pigeons to perform a two-alternative simultaneous discrimination of rotational speed, using stimuli similar to those of Cook et al. (2011). In Experiment 2, we compared relative difficulty of translational speed discrimination and size discrimination in a withinsubject design. Finally, in Experiment 3, we used a two-alternative forced choice task to compare sensitivity to differences in translational speed and size in both pigeons and adult humans.

\section{Experiment 1}

\section{Subjects}

The subjects were four naive, mixed-breed pigeons (Columba livia) purchased as discards from local pigeon breeders. The birds were housed in individual home cages in a well-ventilated room on a 12:12 hr light/dark cycle at Drake University. Grit and water were available ad lib in pigeons' home cages. The birds were maintained at $85 \%$ of their free-feeding body weight by the delivery of millet during experimental sessions and mixed grain after experimental sessions.

\section{Apparatus}

Four custom-built (49 x 49 × $47 \mathrm{~cm}$ ) operant chambers were used in the experiment. One plywood wall of each chamber contained a $32 \times 22-\mathrm{cm}$ opening with a U-shaped track attached to the outside of the wall. The track held a 17-in. infrared touchscreen with an antiglare acrylic filter (CarrolTouch Model \# D27566-001). Pecks to the touchscreen were processed by a USB controller board (CarrolTouch 4000U USB controller, Model \# E053303). A 17-in. LCD Dell monitor (Dell UltraSharp ${ }^{\mathrm{TM}}$ $1708 \mathrm{FP}$, producing $300 \mathrm{~cd} / \mathrm{m}^{2}$ white luminance) was placed directly behind the touchscreen, so that pigeons could see most of the display area. The monitor was set to a 1280 x 1024 -px resolution.

A second plywood wall opposite the touchscreen contained a $7.6 \times 7.6-\mathrm{cm}$ opening to accommodate a grain hopper (MedAssociates Model \# ENV-205M) located outside the operant chamber. A houselight mounted above the grain hopper provided illumination during the experimental sessions. The grain hopper and the houselight were controlled via a relay board (NuDAQ® PCI-7250, Model \# DAQ144). Millet grains served as the food reinforcer.

The relay board and stimulus presentations were controlled by four Dell computers (Optiplex 330 desktop, Intel® Pentium ${ }^{\circledR}$ Dual Core Processor E2160 @ 1.80GHz, 2GB DDR2 SDRAM, integrated video card Intel ${ }^{\circledR}$ GMA3100). A separate Dell computer controlled each individual computer via TightVNC application (retrieved from www.tightvnc.com). All experimental procedures were developed in MATLAB® using functions provided by Psychtoolbox (Brainard, 1997; Pelli, 1997). 


\section{Stimuli}

All stimuli were created in Lightwave 3D, Version 9 (NewTek, Inc., San Antonio, TX), rendered at the rate of $30 \mathrm{fps}$, and saved as QuickTime movies. Each movie (measured 7.9 x $7.9 \mathrm{~cm}$ ) involved a single three-dimensional object (a grey torus) rotating along their $\mathrm{y}$-axis at a set speed. The objects were set upon a blue (stimulus set 1) or green (stimulus set 2) background. In stimulus set 1, S1 rotated at a rate of $4^{\circ}$ per frame or $120^{\circ}$ per second; S2 rotated at $5^{\circ}$ per frame or $150^{\circ}$ per second; and, S3 rotated at $6^{\circ}$ per frame or $180^{\circ}$ per second. In stimulus set $2, \mathrm{~S} 1$ rotated at a rate of $3^{\circ}$ per frame or $90^{\circ}$ per second; S2 rotated at $5^{\circ}$ per frame or $150^{\circ}$ per second; and, S3 rotated at $7^{\circ}$ per frame or $210^{\circ}$ per second. The exact number of frames in the video depended upon the speed of rotation, with as many as needed for the object to complete two 360 degree rotations. The videos were played in a loop until the bird selected one of them. All stimuli in this and subsequent experiments are available at http://www.copal-lab.com/lazarevashare.html.

\section{Procedure}

A trial began with a presentation of an orienting stimulus (a black cross on a white square). After one peck at the square, a pigeon was presented with the two video stimuli $(7.9 \times 7.9 \mathrm{~cm}$ each) for a fixed interval (FI) of $5 \mathrm{~s}$. The left-right location of the stimuli was counterbalanced across trials. The video stimuli were preloaded from previously created Quicktime files at the beginning of each trial and then displayed at the same frame rate as the original video stimuli within MATLAB environment (see Brainard, 1997, for more details). This procedure, as well as Psychtoolbox functions used to display the video information, ensured synchronous presentation of the two videos as well as synchronization of movie frames with the refresh of the monitor.

Once the FI elapsed, the pigeon had to make a single choice peck to one of the two video stimuli. If the correct video was chosen, then the pigeon received $6 \mathrm{~s}$ access to grain and proceeded to an intertrial interval (ITI). The ITI gradually increased from 5 to $20 \mathrm{~s}$ over the course of training to discourage intertrial interference. If the pigeon made an incorrect choice, then the houselight was turned off for the duration of the ITI; these ITIs gradually increased from 5 to $25 \mathrm{~s}$ to make mistakes more punishing and were adjusted on a bird-by-bird basis. The same trial was then repeated until the bird made the correct choice. The correction trials were not used in data analyses.

The pigeons were first trained to discriminate the pairs S1-S2 and S2-S3 using stimulus set 1. A daily training session consisted of 30 blocks of 4 trials ( 2 pairs with left-right location counterbalanced), for a total of 120 trials. For two of the birds, the slower stimulus in each pair was correct (i.e., S1 in the pair S1-S2 and S2 in the pair S2-S3); these contingencies were reversed for the other two birds. We kept the same contingencies across the stimulus sets; if the bird was trained to respond to the slower stimulus in the set 1 , it was also trained to respond to the slower stimulus in the set 2 , and vice versa.

The stimulus set 1 training continued for 14 days. After that, the birds were trained to discriminate a single pair S1-S2 and S2-S3 using stimulus set 2; the background of the stimulus set 2 was changed from blue to green to minimize any potential carryover. This two-pair stimulus set 2 training was presented for 4 days, with the exception of a single bird that received only three days of training due to experimental error. Finally, a single pair (S1-S2) from stimulus set 2 was presented for four additional days. The session structure for both types of stimulus set 2 training was identical to that of stimulus set 1 .

Statistical analyses. All statistical analyses were conducted using multilevel generalized linear modeling (Pinheiro \& Bates, 2000) allowing the use of repeated measures regression with binomial distribution that is more appropriate for our dichotomized choice data than the traditional conversion into proportions (Dixon, 2008; Young, Clark, Goffus, \& Hoane, 2009). Session variable was treated as a continuous predictor, centered to avoid multicollinearity, and log-transformed. Each best fitting model included fixed effects (independent variables similar to those in the traditional analyses) and random effects (within-subject variables that allow for variability across subjects). Therefore, a within-subject 
variable could potentially have both a fixed effect (indicating general influence across all subjects) and a random effect (reflecting individual differences among subjects).

To select the best-fitting model, we first determined the most appropriate random effect structure by comparing a model that included a random effect of subject with the models that added a random effect of pair, session, and pair x session interaction. We used Akaike information criterion (AIC; Akaike, 1974) to select the best random effect structure. After that, we compared this best-fitting random effect model to the models that also included fixed effects of pair, session, and pair x session interaction, again using AIC. Because binomial distribution was chosen, the analysis returned $z$-values, rather than $t$-values. All analyses were conducted in R (R Core Team, 2014) using lme4 package (Bates, Maechler, Bolker, \& Walker, 2015).

\section{Results and Discussion}

Figure 1 (top panel) shows the percent of correct responses to each of the training pairs in stimulus set 1 across 14 training sessions. Unlike Cook et al. (2011) who reported a reliable discrimination after as little as six training sessions (or 480 trials), we have observed no noticeable learning across two weeks of training (a total of 1,680 trials). Importantly, the rotational difference between our stimuli, and therefore their discriminability, was comparable to that in Cook et al.'s (2011) study. At the end of the training, pigeons' average performance to the pair S1-S2 was $54.3 \%, 95 \%$ CI $[51.4,59.4]$; their performance to the pair S2-S3 was $56.7 \%, 95 \%$ CI $[53.6,59.7]$.

The best-fitting model included a fixed effect of pair indicating that the average performance to the pair S2-S3 was slightly better than to the pair S1-S2, $z=2.27, p=0.02$. More importantly, the model did not include a fixed effect of session, confirming the absence of reliable improvement across training sessions at the group level. Finally, the model included a random effect of session indicating individual variability in performance from session to session.

In stimulus set 2, we have increased the discriminability of the training stimuli: The rotational rate of the slow and the fast stimuli in a pair has now differed by $60^{\circ}$ per second instead of $30^{\circ}$ per second as in set 1 . However, as can be seen on Figure 1 (bottom panel) this change had very little effect on pigeons' accuracy. At the end of four training sessions, the birds averaged 46.7\%, 95\% CI [24.8, 68.6] to the pair S1-S2 and 53.3\%, 95\% CI [31.4, 75.2] to the pair S2-S3; neither the fixed effect of session nor the fixed effect of pair were retained in the best-fitting model indicating the lack of consistent effect at the group level.

Unlike Cook et al.'s (2011) study, our design used two pairs of discriminative stimuli presented within a single session. It was therefore possible that the birds would have shown better acquisition if given a single discriminative pair at a time. However, when only one pair, S1-S2 was presented for four consecutive sessions (Figure 2, bottom panel), we have again observed no improvement, with the Session 4 average accuracy equaling to $48.8 \%, 95 \%$ CI $[42.0,55.5]$. The final model again did not include the fixed effect of session, indicating no consistent improvement in accuracy.

Overall, we found that pigeons were unable to quickly learn to simultaneously discriminate two stimuli rotating at different speeds in an experimental procedure that produced rapid and robust acquisition of size discrimination within a comparable time period (Lazareva, Wasserman, \& Young, 2005). We have used discriminative stimuli that were similar to those in Cook et al.'s (2011) report who reported rapid and robust acquisition of a rotational speed discrimination in a go/no-go task. However, unlike a go/no-go discrimination, simultaneous discrimination of two rotating stimuli may have required attending to both stimuli before making a choice. Instead, pigeons may have been concentrating on a single discriminative stimulus or a single stimulus dimension, a strategy that has been reported for other tasks (e.g., Hall, 1974; Lea, Lohman, \& Ryan, 1993; Lea, Wills, \& Ryan, 2006). We therefore redesigned the discriminative task to encourage attending to both video stimuli prior to choice response. 

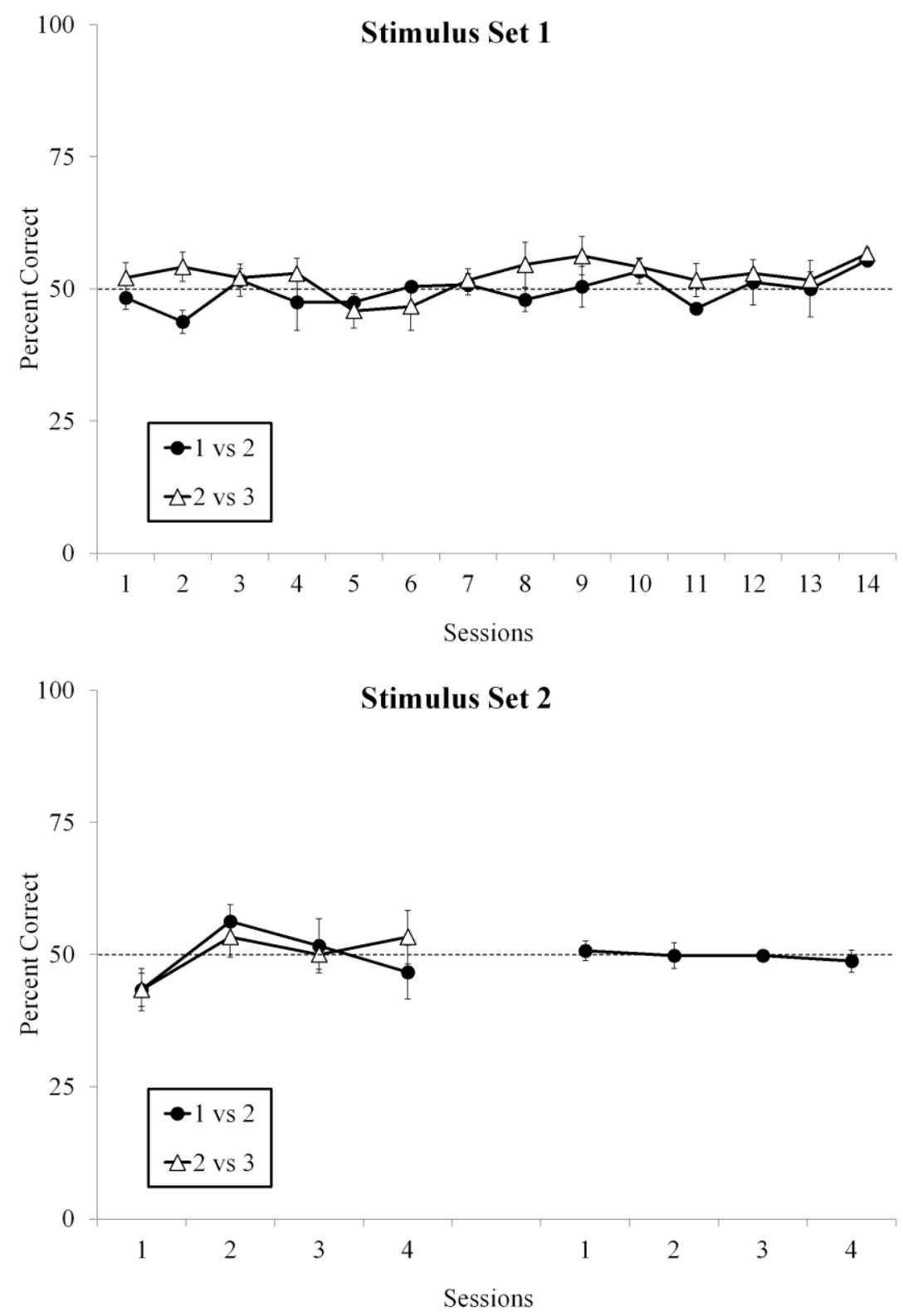

Figure 1. Percent of correct responses to the pairs S1-S2 and S2-S3 using stimulus set 1 (top panel) and stimulus set 2 (bottom panel) in Experiment 1 (rotational motion). Error bars represent standard error of means.

We have also chosen to use translational velocity instead of rotational velocity to further simplify the discrimination. Rotational motion represents the purest form of motion that does not involve any spatial, motion-independent cues such as spatial displacement of an object or differences in motion trajectory. However, this lack of motion-independent cues could have also contributed to slow acquisition in our pigeons ( $c f$ Figure 1). Therefore, we have chosen to use objects moving along y-axis at different speeds as our discriminative stimuli in subsequent experiments.

Our preliminary research has shown that pigeons can indeed learn this type of translational velocity discrimination (see Lazareva, et al., 2014). We have also reported that pigeons appeared to learn size discrimination faster than speed discrimination. However, this earlier report was based on crossexperiment comparison that involved the experiments conducted at different institutions using different operant chambers, different programming languages, different operating systems controlling the operant 
chamber hardware, and different reinforcers. Thus, these procedural disparities could have been responsible for the observed results, instead of the nature of the discriminative stimuli. Here, we have compared relative difficulty of translational speed discrimination and size discrimination in a withinsubject design, to obtain a better estimate of a relative difficulty of both tasks.

\section{Experiment 2}

\section{Subjects and Apparatus}

The subjects were four naive, mixed-breed pigeons (Columba livia) housed and maintained as in Experiment 1. The same apparatus was used as in Experiment 1.

\section{Stimuli}

The motion stimuli were created in Lightwave 3D, Version 9 (NewTek, Inc., San Antonio, TX), rendered at the rate of $30 \mathrm{fps}$ and saved as QuickTime movies. The eight videos involved a blue, threedimensional polyhedron $(1 \mathrm{~cm} \mathrm{x} 1 \mathrm{~cm})$ on a light grey, square background $(7.9 \mathrm{~cm} \times 7.9 \mathrm{~cm})$ moving along the y-axis at different speeds. The slowest stimulus, S1, moved at a speed of $0.08 \mathrm{~cm}$ per frame, with a constant increment in speed of approximately $0.10 \mathrm{~cm}$ per frame from one stimulus to the next. Each movie contained as many frames as necessary to complete the motion from the bottom of the screen to the top and back to the bottom; thus, the number of frames ranged from 90 (S1) to 11 (S7). During a trial, all of the videos were played in a loop until the bird made a choice.

The size stimuli were four white circles drawn by using Psychtoolbox function. The diameter of the smallest circle was $30 \mathrm{px}$, with a constant increment of $10 \mathrm{px}$ from one stimulus to the next. Two pairs of stimuli, S1-S2 and S6-S7 (where numbers stand for increasing size or speed of the stimulus) were selected for training among the size stimuli and the motion stimuli. We have expected that the birds will learn S1-S2 discrimination faster than S6-S7 discrimination as constant increments along the sensory dimension are not equally discriminable (Stevens, 1957). The exact values of the size stimuli and the motion stimuli are shown in Table 1.

Table 1

Values of stimuli along size and speed dimension in Experiment 2

\begin{tabular}{ccccc}
\hline \multirow{2}{*}{ Stimulus } & \multicolumn{2}{c}{ Circle diameter } & \multicolumn{2}{c}{ Speed of motion } \\
\cline { 2 - 5 } & in px & in cm & in px per s & in cm per s \\
\hline S1 & 30 & 0.79 & 93 & 2.4 \\
S2 & 40 & 1.05 & 198 & 5.1 \\
S6 & 80 & 2.11 & 645 & 17.1 \\
S7 & 90 & 2.37 & 765 & 20.1 \\
\hline
\end{tabular}

\section{Procedure}

All birds were trained to perform the size discrimination task and the speed discrimination task for 20 consecutive, completed training sessions, with the order of the tasks counterbalanced across the birds. For two birds, both the smaller circle and the slower object were correct; these contingencies were reversed for the other two birds. All training sessions consisted of 30 blocks of four trials (two pairs with left-right location counterbalanced), for a total of 120 trials.

Training: Size discrimination. Figure 2 (left panel) illustrates the trial sequence in the size discrimination task. A trial began with a presentation of an orienting stimulus (a black cross on a white square). After one peck at the square, a pigeon was presented with the two white circles drawn on the 
black background for a FI of $5 \mathrm{~s}$. Because the sizes of the circles differed ( $c f$ Table 1), a peck within either of the two $3.5 \times 3.5-\mathrm{cm}$ square areas aligned with the circles advanced the trial. The stimulus to which the response had been made remained on for $1 \mathrm{~s}$ while the other stimulus disappeared.

If the choice was correct, then the bird received $6 \mathrm{~s}$ access to grain and proceeded to the ITI. The ITI gradually changed from 10 to $15 \mathrm{~s}$ on bird-by-bird basis to reduce intertrial interference. If the pigeon made an incorrect choice, then the houselight was turned off for the duration of the ITI; these ITIs gradually increased from 5 to $20 \mathrm{~s}$ to make mistakes more punishing. The same trial was then repeated until the bird made the correct choice. The correction trials as well as occasional incomplete sessions were not used in the data analyses.

\section{Size discrimination}

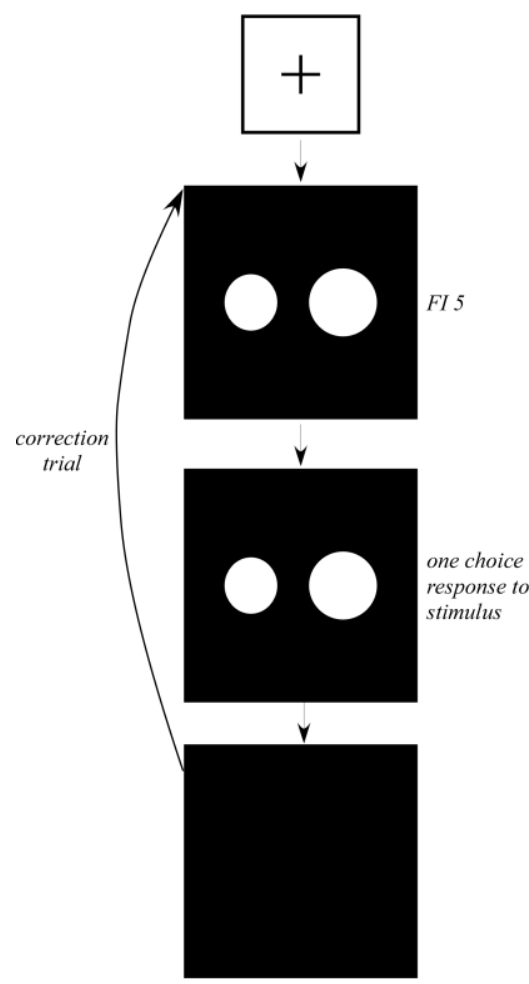

\section{Speed discrimination}

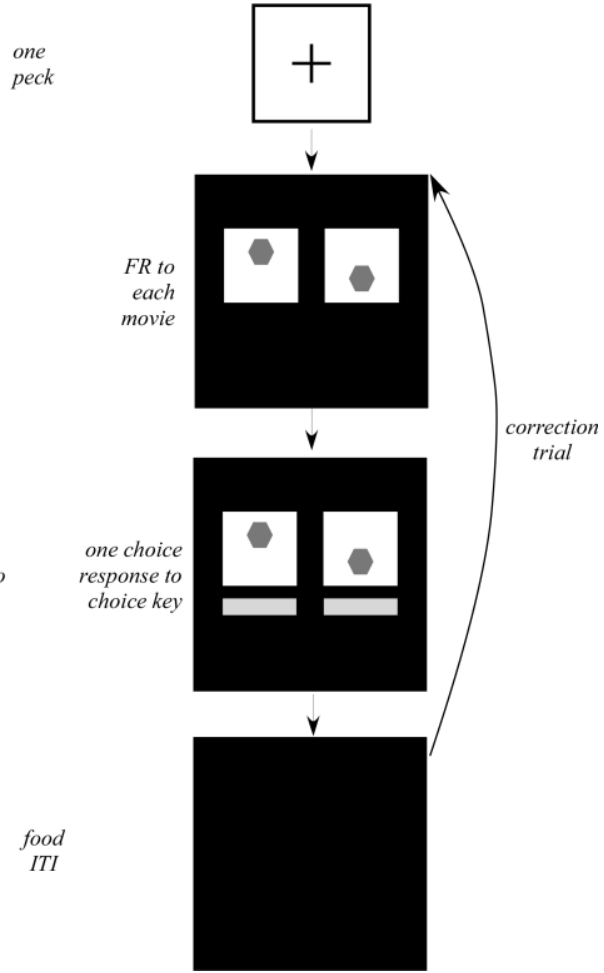

Figure 2. The sequence of events in a two-alternative simultaneous choice task for size discrimination (left panel) and speed discrimination (right panel) in Experiment 2.

Training: Speed discrimination. Figure 2 (right panel) illustrates the trial sequence in the speed discrimination task. After a single response to the orienting stimulus, the bird was shown two video stimuli $(7.9 \times 7.9 \mathrm{~cm}$ each) presented as described in Experiment 1.

Next, the birds had to complete a fixed ratio (FR) requirement to each of the video stimuli; for example, the FR 4 required that the bird pecked each video four times, for the total of eight responses. The FR was gradually increased from one to four responses, in order to encourage the birds to attend to both videos.

Once the FR was completed, two green response buttons $(1.3 \times 7.9 \mathrm{~cm})$ appeared under each video stimulus, and the bird had to make a single choice response to one of the buttons. If the button under the correct video stimulus was chosen, then the bird received $6 \mathrm{~s}$ access to grain and proceeded to the ITI. The ITI gradually changed from 10 to $15 \mathrm{~s}$ on bird-by-bird basis to reduce intertrial interference. If the pigeon made an incorrect choice, then the houselight was turned off for the duration of the ITI; these ITIs gradually increased from 5 to $28 \mathrm{~s}$ to make mistakes more punishing. The same trial was then 
repeated until the bird made the correct choice. The correction trials as well as occasional incomplete sessions were not used in the data analyses.

Statistical analyses. All statistical analyses were conducted using multilevel generalized linear modeling following the same approach as in Experiment 1.

\section{Results and Discussion}

Figure 3 (top panel) shows the average accuracy in size discrimination task separately for the pairs S1-S2 and S6-S7. At the beginning of the training, the accuracy to both pairs was not significantly different from chance (M and 95\% CI for S1-S2: 48.8\%, [29.6, 67.9]; for S6-S7: 46.3\%, [33.9, 58.6]; both $t$ 's $<1$ ). However, by the end of the training, both pairs were significantly above chance (S1-S2: 95.4\%, $[90.4,100.0], t(3)=28.79, p<0.0001, d=14.41$; S6-S7: 64.6\%, [56.4, 72.8], $t(3)=5.65, p=0.01, d=$ 2.83). As expected, the birds were noticeably faster and more accurate at learning S1-S2 discrimination than at learning S6-S7 discrimination, indicating a decrease in subjective discriminability with an increase in size of the stimuli.

\section{Size Discrimination}
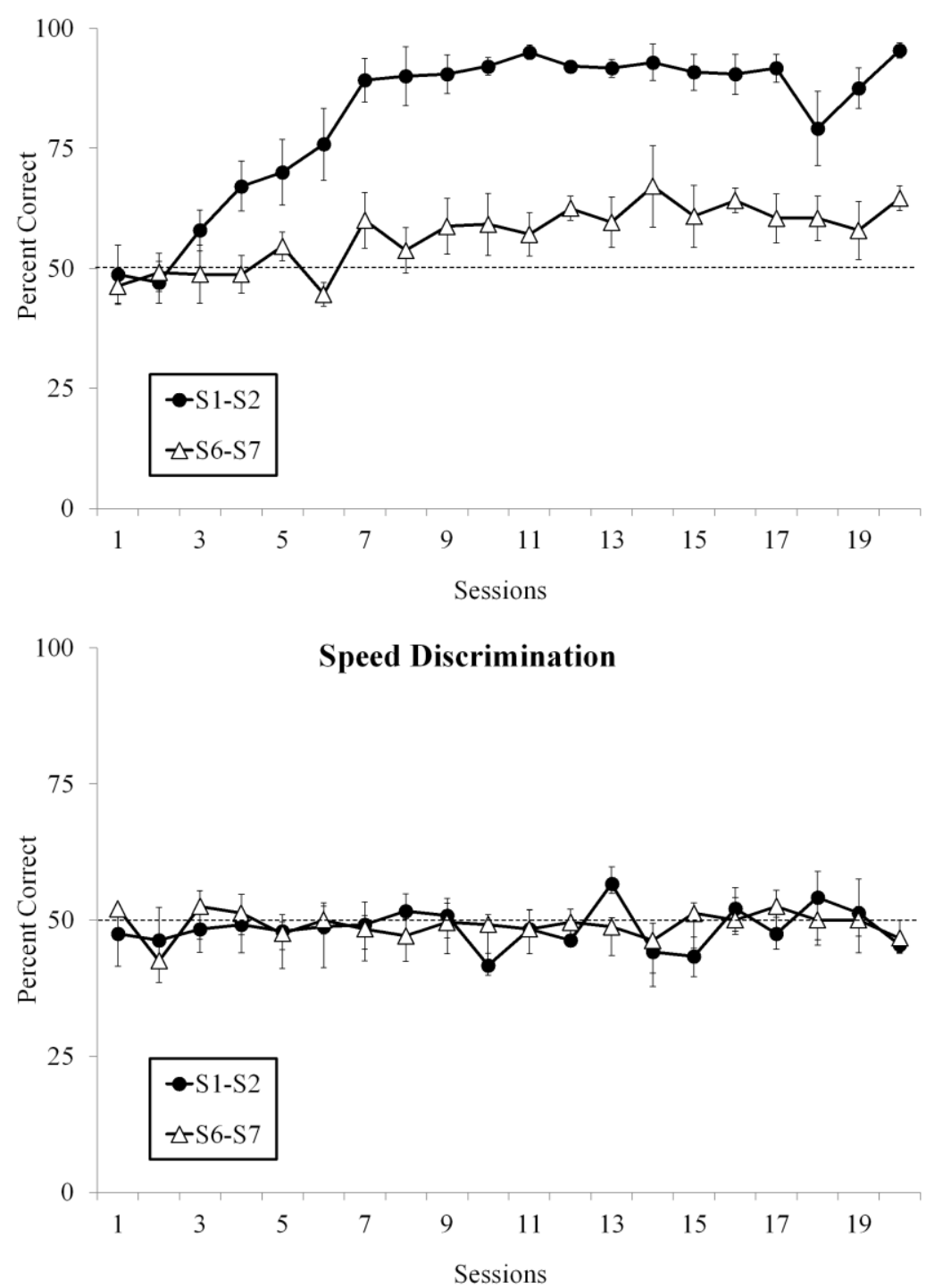

Figure 3. Percent of correct responses to the pairs S1-S2 and S6-S7 in size discrimination task (top panel) and speed discrimination task (bottom panel) in Experiment 2. Error bars represent standard error of means. 
In contrast, as Figure 3 (bottom panel) shows, speed discrimination did not rise significantly above chance for either pair by the end of the training (S1-S2: 45.4\%, [39.9, 50.9], $t(3)=-2.67, p=0.076$, $d=-1.35$; S6-S7: 46.7\%, [37.7, 55.6], $t(3)=-1.19, p=0.302, d=-0.59)$. In other words, despite an extensive training the birds showed little noticeable improvement even with the "easy" S1-S2 discrimination.

Preliminary analyses with pair, session, and training type as fixed effects found a significant three-way interaction, $z=7.16, p<0.0001$. To simplify the interpretation of the results, we conducted two separate analyses for each training condition. For size discrimination, the best-fitting model included both fixed and random effects of session, indicating a consistent increase in average accuracy from the first to the last day of training, $z=12.35, p<0.0001$, despite the presence of individual differences. The model also included both fixed and random effects of pair, indicating that accuracy to the pair S1-S2 was consistently higher than the accuracy to the pair S2-S3, $z=-9.49, p<0.0001$. Finally, the model also included a pair $\mathrm{x}$ session interaction, $z=-11.15, p<0.0001$, demonstrating that the performance to the pair S1-S2 improved more rapidly than the performance to the pair S6-S7. In contrast, the same modeling approach applied to speed discrimination produced the best-fitting model that did not include fixed or random effects of session or pair, indicating that accuracy did not appear to improve across training sessions and was equivalent for both training pairs for all birds.

In summary, we found that pigeons were significantly more accurate performing size discrimination than speed discrimination. Moreover, the birds found the pair S1-S2 to be easier than the pair S6-S7 when discriminating sizes, but not when discriminating speeds, suggesting a possible lower sensitivity to differences in speed than to differences in size. This possibility was explored in the next experiment.

\section{Experiment 3}

In Experiment 3, we directly explored pigeons' sensitivity to differences in speed versus differences in size by training them to discriminate two extreme values and then testing their discriminative performance with intermediate values in the two-alternative forced-choice task. We also conducted a similar experiment with human participants for comparative purposes.

\section{Subjects}

The subjects were six naive, mixed-breed pigeons (Columba livia) obtained and housed as in Experiment 1 . The participants were eight undergraduate students ( 2 male, 6 female) at Drake University who participated in the experiment for course credit during the academic year.

\section{Apparatus and Stimuli}

The same apparatus as in Experiments 1 and 2 was used for pigeons. The human participants were tested using four Apple ${ }^{\circledR}$ iMac ${ }^{\circledR}$ computers with 21.5" displays located in a single testing room. The presentation of all stimuli for human participants was programmed in Superlab, Version 5.0 (Cedrus Corporation, San Pedro, CA).

Two sets of discriminative stimuli were used, one differing in size and another differing in speed. The stimuli S1 and S12 were used as training stimuli whereas the stimuli S2 through S11 were used during testing. All stimuli were prepared as described in Experiment 2. Table 2 lists sizes and speeds for both sets of stimuli. 
Table 2

Values of training stimuli (S1 and S12) and testing stimuli (S2 through S11) along size and speed dimension in Experiment 3

\begin{tabular}{ccccc}
\hline \multirow{2}{*}{ Stimulus } & \multicolumn{2}{c}{ Circle diameter } & \multicolumn{2}{c}{ Speed of motion } \\
\cline { 2 - 5 } & in px & in cm & in px per s & in cm per s \\
\hline S1 & 30 & 0.79 & 93 & 2.4 \\
S2 & 32 & 0.84 & 108 & 2.7 \\
S3 & 35 & 0.92 & 132 & 4.2 \\
S4 & 39 & 1.03 & 162 & 5.1 \\
S5 & 43 & 1.13 & 195 & 6.3 \\
S6 & 48 & 1.27 & 237 & 7.5 \\
S7 & 53 & 1.4 & 288 & 9.3 \\
S8 & 59 & 1.56 & 351 & 11.1 \\
S9 & 66 & 1.74 & 426 & 13.5 \\
S10 & 73 & 1.92 & 516 & 16.5 \\
S11 & 81 & 2.14 & 630 & 20.1 \\
S12 & 90 & 2.37 & 765 & \\
\hline
\end{tabular}

Procedure

The order of discriminative tasks (size or speed) as well as the response assignment (e.g., left key for smaller size for human participants) was counterbalanced across subjects.

Pigeons. Instead of two-alternative simultaneous discrimination, pigeons were trained to perform a two-alternative forced-choice task. At the beginning of the trial, a white square was presented and a bird had to respond to it once to initiate the trial. After that, a single stimulus was presented until the bird completed an FR requirement. The FR was gradually increased from 1 to 13 pecks on a bird-by-bird basis to improve attention to the stimulus and to make errors more costly. Once the FR was completed, two response buttons appeared on the left and the right sides of the stimulus display and the bird had to select one of them. The assignment of response buttons to the choices (e.g., left button is correct for a slow stimulus) was counterbalanced across birds.

If the bird selected the correct response button, then the food was made available for $6 \mathrm{~s}$ and a 10$\mathrm{s}$ ITI ensued. If the choice was incorrect, then the houselight was turned off for the duration of the ITI; these ITIs gradually increased from $5 \mathrm{~s}$ up to $29 \mathrm{~s}$ to make errors more punishing. After that, the bird had to repeat the same trial until it made the correct choice. These correction trials were recorded but not used in any analyses.

Each training session consisted of 20 blocks of 6 trials ( 3 trials x 2 stimuli), for a total of 120 trials. Once pigeons reached $80 \%$ correct choices during a single training session, they were exposed to testing. Each testing session began with 2 warm-up blocks of 6 training trials; these trials were excluded from all analyses. After that, the birds were exposed to 20 blocks of 7 trials ( 6 training trials plus 1 testing trial), so that at the end of the testing session the bird received 2 presentations of each testing stimulus (S2 through S11). The performance criterion had to be maintained during testing; if the bird's performance deteriorated, then it was returned to training until it reached the criterion. Testing continued until each bird completed 15 testing sessions, for the total of 30 trials with each testing stimulus.

Human participants. Prior to the experiment, the participants were informed that they would be viewing visual stimuli on the screen and that their task was to press a key on a computer keyboard in response to the stimulus. The participants were not explicitly instructed to perform a fast/slow or a small/large discrimination; instead, they were told that their task was to achieve as many correct responses as possible. Participants sat without head restraints approximately $55 \mathrm{~cm}$ from the monitor.

The trial began with an orienting display presented for $1000 \mathrm{~ms}$; the display showed the trial number and the accumulated number of the correct responses. Next, the participants were presented with a single discriminative stimulus located in the middle of the computer monitor until they pressed " $\mathrm{X}$ " or 
"Z" on the computer keyboard. The correct response was followed by a presentation of a word "Correct!" for $900 \mathrm{~ms}$, and a presentation of the next trial after $500 \mathrm{~ms}$ ITI. The orienting display on the next trial showed an increased number of correct responses, providing an additional feedback for the participants. The incorrect response was followed by a presentation of a word "Incorrect!" for $900 \mathrm{~ms}$ and a return to the same trial (correction trial) until the correct response has been made. The orienting display following the correction trial did not show an increased number of correct responses indicating an error.

The experimental session began with the presentation of blocks of 8 training trials ( 4 trials with S1 and 4 trials with S12), until the participants made 10 consecutive correct responses. After that, they were presented with 10 blocks of testing trials; however, the participants received no explicit notification of the change in the nature of the experiment. Each testing block contained 10 training trials and 10 testing trials (1 trial with the stimuli S2 through S11) so that by the end of the testing they received 10 presentations for each novel, testing trial. The participants continued to receive differential feedback on training trials; however, on testing trials any response was followed by "Correct!" and an increment in the number of correct responses on the next trial's orienting display.

Once the first assigned task has been completed, the participants viewed a message that instructed them to take a break before proceeding to the next task. After the second task, the participants were fully debriefed, thanked for their participation, and permitted to leave.

\section{Statistical Analyses}

All statistical analyses were conducted using multilevel generalized nonlinear modeling following the same approach as in Experiment 1. Stimulus was treated as a continuous predictor and centered to avoid multicollinearity. The derived function graphs (Figure 4) were created by using lattice package in $\mathrm{R}$ (Sarkar, 2008). The bootstrapped confidence intervals were calculated using $\mathrm{BC}_{\mathrm{a}}$ method with 1000 replicates from boot package in R (Canty \& Ripley, 2014).

\section{Results and Discussion}

On average, pigeons required fewer sessions to reach training criterion in the size task than in the speed task (M and 95\% CI for size: 3.2, [2.7, 3.6]; for speed: 11.7, [3.9, 19.5]; two-tailed paired t-test, $t(5)$ $=-2.75, p=0.04, d=-0.72$ ). Similarly, human participants also required fewer trials to reach criterion in the size task than in the speed task ( $\mathrm{M}$ and 95\% $\mathrm{CI}$ for size: 15.5, [9.4, 21.6]; for speed: 27.0, [17.3, 36.7]; two-tailed paired t-test, $t(7)=-3.29, p=0.01, d=-1.17$ ). This difference suggested that both pigeons and human participants found initial size discrimination to be easier than speed discrimination.

Figure 4 presents the derived functions separately for size discrimination and speed discrimination for pigeons (left panel) and people (right panel), with both species clearly demonstrating steeper slopes in size discrimination task than in speed discrimination task. The best-fitting model for pigeon data included random effects of stimulus and training type (speed, size) as well as their interaction, indicating individual differences in the effect of these variables on pigeons' accuracy. More importantly, the model included the fixed effects of stimulus type, $z=55.4, p<0.0001$, and training type, $z=-2.1, p=$ 0.036 , as well as training type $\mathrm{x}$ stimulus type interaction, $z=5.01, p<0.0001$, indicating that these variables had a consistent effect at the group level. The estimated mean slope was $0.59,95 \%$ CI [0.57, $0.61]$ for size discrimination task and $0.43,95 \%$ CI [0.38, 0.47] for speed discrimination task confirming higher sensitivity to size differences than to speed differences observed in previous experiments.

The best-fitting model for human data included random effects of stimulus and training type as well as their interaction, just like it did for pigeons. Although the fixed effect of training type failed to reach significance, $z=-0.99, p=0.322$, both the fixed effect of stimulus type, $z=6.62, p<0.0001$, and stimulus type $\mathrm{x}$ training type interaction, $z=2.56, p=0.01$, were significant. The estimated mean slope was $1.03,95 \%$ CI $[0.71,1.34]$ for size discrimination task and $0.65,95 \%$ CI $[0.52,0.86]$ for speed discrimination task indicating that just like pigeons, human participants appeared to be more sensitive to size differences than to speed differences. 

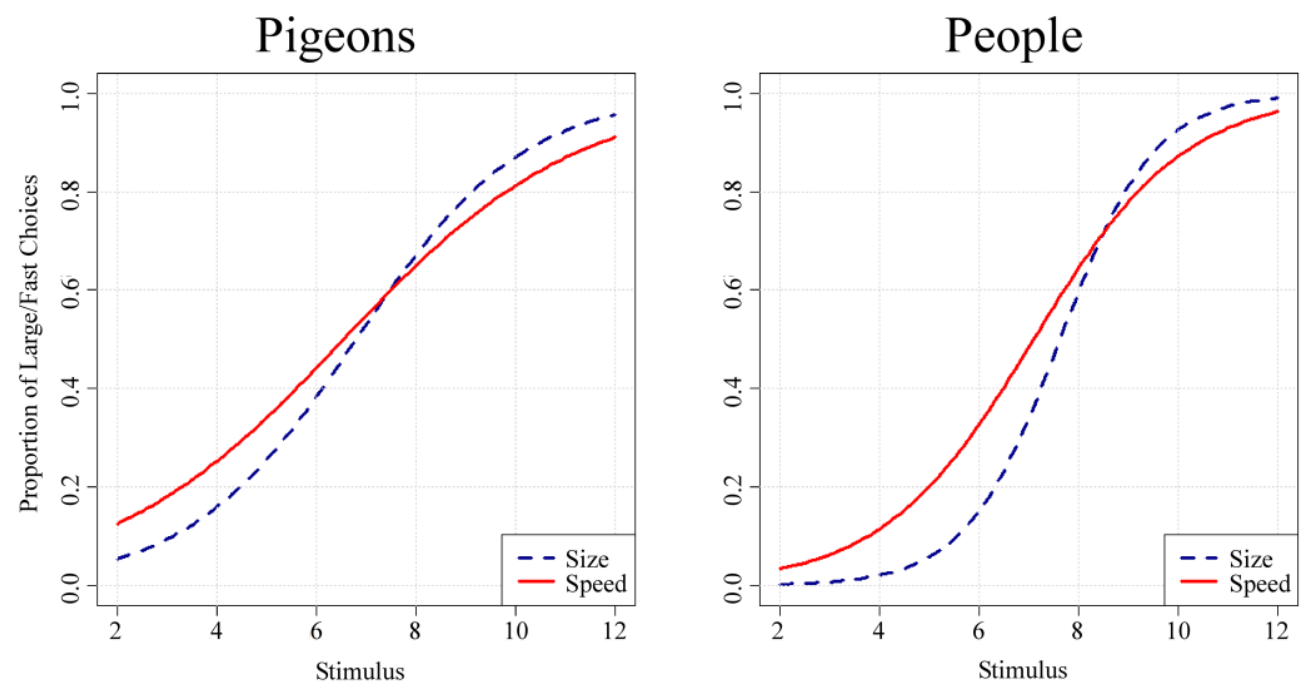

Figure 4. Multilevel logistic regression fits of the proportion of larger/faster choices in size discrimination task and speed discrimination task for each stimulus value for pigeons (left) and human participants (right).

\section{General Discussion}

Earlier, we reported that pigeons appeared to easily acquire simultaneous two-alternative size discrimination but not speed discrimination (Lazareva et al., 2014). In Experiment 1, we found no observable improvement in discrimination of rotational speed in a simultaneous two-alternative task after an extensive training. In Experiment 2, pigeons showed no improvement in discrimination of translational speed by the end of the training period that was sufficient to produce an asymptotic performance in size discrimination task ( $c f$ Figure 3). In Experiment 3, we found that pigeons appeared to be more sensitive to the differences in size than to the differences in speed in a two-alternative forced-choice task; the same was true for human participants ( $c f$ Figure 4).

Overall, our results are not consistent with many earlier reports of motion discrimination in pigeons that suggested high sensitivity to object speed (Cook et al., 2011; Herbranson et al., 2002; Koban \& Cook, 2009; but see Hodos, Smith, \& Bonbright, 1976; Mulvanny, 1978). Importantly, the magnitude of the differences among the stimuli in our study was comparable to the previously used magnitudes. For example, Cook et al. (2011) reported generalization decrement when a discriminative stimulus rotated in depth $30^{\circ}$ faster than the previously trained S+ suggesting that this difference was discriminable. Yet, pigeons in our Experiment 1 were unable to discriminate two stimuli even when one rotated $60^{\circ}$ faster than another (set 2, cf Figure 1). Similarly, Herbranson et al. (2002) reported a successful categorization of "slow" and "fast" stimuli in which the displacement of the "fast" stimulus was $0.9 \mathrm{~cm} / \mathrm{s}$ greater. Yet, in our Experiment 2 pigeons failed to acquire a discrimination in which the "fast" stimulus displacement was more than $3 \mathrm{~cm} / \mathrm{s}$ greater ( $c f$ Figure 3 ). Thus, the discriminability of our stimuli was comparable to or greater than that reported previously despite the dramatically different outcomes.

Could the differences in the discriminative performance across two different tasks be attributed to procedural disparities rather than to differential sensitivity to stimulus dimensions? The size discrimination task in Experiment 2 used a simple two-alternative simultaneous discrimination procedure in which pigeons were required to peck the correct stimulus once at the end of the fixed interval in order to obtain food. In contrast, during the speed discrimination task, they were required to complete a certain number of pecks to each of the training stimuli and then select a separate response button underneath the correct stimulus. Responding to a separate area of the screen instead of directly to a stimulus is arguably more demanding for a pigeon; thus, it could be suggested that the speed discrimination task was more challenging due to the difficult response requirements rather than due to the stimulus nature. It is worth noting, however, that these different response requirements were introduced after pigeons failed to learn 
speed discrimination in a two-alternative simultaneous discrimination task in Experiment 1, in which the birds were allowed to respond directly to the video stimulus. Moreover, pigeons and human participants in Experiment 3 performed both tasks using an identical procedure (a two-alternative forced-choice task); yet, both species demonstrated slower learning and lower sensitivity to motion dimension in contrast to size dimension. We therefore believe that our results are unlikely to be explained by appealing to the differential difficulty in response requirements.

One could also suggest that our Experiment 3 results could be explained by the range of selected training and testing stimuli for each task. For example, we might have unintentionally selected an artificially wide range of size stimuli and an artificially narrow range of speed stimuli, resulting in a shallower slope for size and a steeper slope for speed. However, our speed and size stimuli had a reasonably wide range and the values comparable to those in prior research (Hodos et al., 1976; Mulvanny, 1978).

A somewhat different but related concern is the values of our chosen endpoint stimuli in Experiment 3. Both pigeons and people were significantly slower to learn the speed discrimination than the size discrimination in Experiment 3 suggesting that the speed task remained somewhat more difficult despite the dramatic differences in the velocities of the discriminative stimuli. However, previous research indicates that the more difficult initial discrimination usually produces higher sensitivity to the stimulus dimension and, as a result, higher discriminative performance to the pairs of the stimuli along this dimension in comparison to the easier discrimination (e.g., Marsh \& Paulson, 1969; Zeiler, 1966). Instead, our more difficult speed discrimination appears to be associated with the lower sensitivity than the easier size discrimination.

Still, it is possible that a choice of different training and testing stimuli would produce a different experimental outcome than the one reported here. Future research should address this concern by employing modern parametric procedures designed for estimating psychometric curves (e.g., Fründ, Haenel, \& Wichmann, 2011).

We propose that the methodological difference responsible for our results is the requirement of the simultaneous comparison of the two discriminative stimuli. The prior experiments reporting successful acquisition of velocity information employed either a go/no-go procedure or a two-alternative forced-choice task. In both of these tasks, pigeons view a single discriminative stimulus instead of explicitly comparing two simultaneously presented stimuli as in our Experiments 1 and 2 . It is then possible that this additional requirement of comparing the two simultaneously presented velocities made the task particularly challenging in comparison to the prior reports.

Why would we observe the relative insensitivity to differences in speed of two simultaneously presented stimuli? On one hand, such insensitivity may be attributable to general properties of visual system; for example, because the size stimuli are static, their comparison would require less time than the comparison of speed stimuli and may therefore be less prone to error. One the other hand, it is possible that both pigeons and humans are more sensitive to differences in size than to differences in speed because of their ecological environment. It seems intuitively plausible that being attuned to fine differences in size can be quite useful in many situations associated with foraging, aggressive interactions, or mate choice. In contrast, non-predatory species may not frequently encounter situations that would necessitate a simultaneous comparison of two speeds. If this latter supposition were correct, then we would expect obligate predatory species such as hawks or wolves to be as sensitive to differences in speed as to differences in size. Future research should explore this interesting possibility.

\section{Acknowledgments}

We would like to thank Gwendolyn Carlson, Emily Leiker, and Tonya Weaver who have contributed to data collection and early stages of data analyses. This research was supported by Drake University Arts and Sciences Faculty Development Fund to OFL. 


\section{References}

Akaike, H. (1974). A new look at the statistical model identification. Automatic Control, IEEE Transactions on, 19, $716-723$

Asen, Y., \& Cook, R. G. (2012). Discrimination and categorization of actions by pigeons. Psychological Science, $23,617-624$.

Bates, D., Maechler, M., Bolker, B., \& Walker, S. (2015). Fitting linear mixed-effects models using lme4. Journal of Statistical Software, 67, 1 - 48.

Brainard, D. H. (1997). The psychophysics toolbox. Spatial Vision, 10, 433 - 436.

Canty, A., \& Ripley, B. (2014). Boot: Bootstrap R (S-Plus) functions [Computer software]. R package version 1.311. Retrieved from https://cran.r-project.org.

Cook, R. G., Beale, K., \& Koban, A. (2011). Velocity-based motion categorization by pigeons. Journal of Experimental Psychology: Animal Behavior Processes, 37, 175 - 188. doi: 10.1037/a0022105

Dixon, P. (2008). Models of accuracy in repeated-measures designs. Journal of Memory and Language, 59,447 456. doi: 10.1016/j.jml.2007.11.004

Fründ, I., Haenel, N. V., \& Wichmann, F. A. (2011). Inference for psychometric functions in the presence of nonstationary behavior. Journal of Vision, 11, 1-19. doi: 10.1167/11.6.16.

Hall, G. (1974). Strategies of simultaneous discrimination learning in the pigeon. The Quarterly Journal of Experimental Psychology, 26, 520 - 529. doi: 10.1080/14640747408400442

Herbranson, W. T., Fremouw, T., \& Shimp, C. P. (2002). Categorizing a moving target in terms of its speed, direction, or both. Journal of Experimental Analysis of Behavior, 78, $249-270$.

Hodos, W., Smith, L., \& Bonbright, J. C. (1976). Detection of the velocity of movement of visual stimuli by pigeons. Journal of the Experimental Analysis of Behavior, 25, 143 - 156. doi: 10.1901/jeab.1976.25-143

Jenkins, W. O., Pascal, G. R., \& Walker, R. W., Jr. (1958). Deprivation and generalization. Journal of Experimental Psychology, 56, 274 - 277. doi: 10.1037/h0043850

Koban, A., \& Cook, R. G. (2009). Rotational object discrimination by pigeons. Journal of Experimental Psychology: Animal Behavior Processes, 35, $250-265$.

Lazareva, O. F., Wasserman, E. A., \& Young, M. E. (2005). Transposition in pigeons: Reassessing Spence (1937) with multiple discrimination training. Learning and Behavior, 33, $22-46$.

Lazareva, O. F., Young, M. E., \& Wasserman, E. A. (2014). A three-component model of relational responding in the transposition paradigm. Journal of Experimental Psychology: Animal Behavior Processes, 40, 63 - 80. doi: 10.1037/xan0000004

Lea, S. E. G., Lohman, A. E., \& Ryan, C. M. (1993). Discrimination of five-dimensional stimuli by pigeons: Limitations of feature analysis. Quarterly Journal of Experimental Psychology, 46B, $19-42$.

Lea, S. E. G., Wills, A. J., \& Ryan, C. M. (2006). Why are artificial polymorphous concepts so hard for birds to learn? Quarterly Journal of Experimental Psychology, 59 , 251 - 267.

Marsh, G., \& Paulson, N. (1969). Transposition as a function of problem difficulty. Journal of Experimental Psychology, 80, $156-159$.

Mulvanny, P. (1978). Velocity discrimination by pigeons. Vision Research, 18, 531 - 536. doi: 10.1016/00426989(78)90198-0

Peissig, J. J., Kirkpatrick-Steger, K., Young, M. E., Wasserman, E. A., \& Biederman, I. (2006). Effects of varying stimulus size on object recognition in pigeons. Journal of Experimental Psychology: Animal Behavior Processes, 32, 419 - 430. doi: 10.1037/0097-7403.32.4.419

Pelli, D. G. (1997). The VideoToolbox software for visual psychophysics: Transforming numbers into movies. Spatial Vision, 10, $437-442$.

Pinheiro, J. C., \& Bates, D. M. (2000). Mixed-effect models in S and S-Plus. New York: Springer-Verlag.

Pisacreta, R., Potter, C., \& Lefave, P. (1984). Matching of varying-size form stimuli in the pigeon. Bulletin of the Psychonomic Society, 22, $591-593$.

R Core Team. (2014). R: A language and environment for statistical computing [Computer software]. R Foundation for Statistical Computing, Vienna, Austria. Retrieved from http://www.R-project.org/.

Sarkar, D. (2008). Lattice: Multivariate data visualization with R. New York: Springer.

Stevens, S. S. (1957). On the psychophysical law. Psychological Review, 64, 153 - 181. doi: 10.1037/h0046162

Wildemann, D. G., \& Holland, J. G. (1973). The effect of the blackout method on acquisition and generalization. Journal of the Experimental Analysis of Behavior, 19, 73 - 80. doi: 10.1901/jeab.1973.19-73

Young, M. E., Clark, M. H., Goffus, A., \& Hoane, M. R. (2009). Mixed effects modeling of Morris water maze data: Advantages and cautionary notes. Learning and Motivation, 40, 160 - 177. 
Zeiler, M. D. (1966). Solution of the two-stimulus transposition problem by four- and five-year-old children. Journal of Experimental Psychology, 71, 576 - 579. 